\title{
Prevalência de transtornos mentais comuns e fatores associados entre idosos de um município do Brasil
}

\author{
The prevalence of common mental disorders and associated factors \\ among the elderly in a Brazilian city
}

\author{
Paloma Alves dos Santos da Silva ${ }^{1}$ \\ Saulo Vasconcelos Rocha ${ }^{1}$ \\ Loiamara Barreto Santos ${ }^{1}$ \\ Clarice Alves dos Santos ${ }^{1}$ \\ Camila Rego Amorim ${ }^{1}$ \\ Alba Benemérita Alves Vilela ${ }^{1}$
}

\footnotetext{
${ }^{1}$ Departamento de Saúde, Universidade Estadual do Sudoeste da Bahia. Av. José Moreira Sobrinho s/n, Jequiezinho. 45200-000 Jequié BA Brasil. paloma_ass@hotmail.com
}

\begin{abstract}
With advancing age, the presence of psychic morbidities is more frequent and jeopardizes the quality of life of the population. The scope of this study was to estimate the prevalence and factors associated with Common Mental Disorders (CMD) in the elderly population resident in a municipality. It involved a cross-sectional study with 310 elderly people residing in Ibicui in the State of Bahia. A questionnaire assessing sociodemographic characteristics, lifestyle, health status and screening for CMD (Self-Reporting Questionnaire - SRQ-20) was used. For statistical analysis, Poisson regression was used, with calculation of prevalence ratios, confidence intervals (95\%) and $p \leq 0.05$ significance level. The general prevalence of CMD was $55.8 \%$. The female individuals who reported rheumatism presented higher prevalence of CMD. It is recommended that prevention and control of these morbidities among the elderly population of the municipality should be implemented.
\end{abstract}

Key words Elderly, Health of the elderly, Mental disorders
Resumo Com o avançar da idade, a presença de morbidades psíquicas são mais frequentes $e$ comprometem a qualidade de vida das pessoas. $O$ objetivo do presente estudo foi estimar a prevalência e os fatores associados a Transtornos Mentais Comuns (TMC) na população idosa residente em um município brasileiro. Estudo transversal com 310 idosos, em Ibicuí-BA. Foi utilizado formulário com características sociodemográficas, hábitos de vida, estado de saúde e triagem para $T M C$ (Self-Reporting Questionnaire - SRQ-20). Para análise estatística, foi utilizada a regressão de Poisson, com cálculo das razões de prevalência, intervalo de confiança (95\%) e nivel de significância $p \leq 0,05$. A prevalência geral de TMC foi de $55,8 \%$. Os indivíduos do sexo feminino e que referiram reumatismo apresentaram maior prevalência de TMC. Recomenda-se a realização de ações de prevenção e controle dessas morbidades entre a população idosa do município.

Palavras-chave Idoso, Saúde do idoso, Transtornos mentais 


\section{Introdução}

Os transtornos mentais representam aproximadamente $12 \%$ da carga total de doenças ${ }^{1,2}$. Dentre estes, Transtornos Mentais Comuns (TMC) que se caracterizam por um conjunto de sintomas incluindo ansiedade, insônia, fadiga, irritabilidade, esquecimento, dificuldade de concentração e queixas somáticas ${ }^{3}$, apresentam-se como uma das morbidades psíquicas mais prevalentes, atingindo aproximadamente um terço da população em indivíduos de diferentes faixas etárias ${ }^{4,5}$.

Embora não sejam tão graves como distúrbios psicóticos, TMC podem representar um importante problema de saúde pública devido à sua alta prevalência e graves efeitos sobre o bem - estar pessoal, familiar, trabalho e uso de serviços de saúde ${ }^{6}$.

Estudos internacionais mostram prevalências de TMC variando entre $32,4 \%$, na Etiópia, a $51,8 \%$, na Dinamaca ${ }^{6,7}$. No Brasil, esta prevalência varia entre $29,6 \%$ a $47,4 \%{ }^{8,9}$. Indivíduos de idade avançada, do sexo feminino, de baixa renda, baixo nível de escolaridade, tabagistas ${ }^{9,10}$, divorciados ou viúvos ${ }^{9}$, de cor negra ou parda e doentes $\operatorname{crônicos}^{10}$, são os que apresentam maiores prevalências de TMC.

Entre os idosos, a prevalência observada de TMC varia de 29,7\%, no município de Campinas - $\mathrm{SP}^{11}$ a $47,7 \%$, em Jequié-BA . Situações como abandono, isolamento social, incapacidade de retorno à atividade produtiva entre outros, são fatores que comprometem a qualidade de vida e podem aumentar a exposição dos idosos às morbidades psíquicas ${ }^{12}$.

Apesar da crescente produção científica na área do envelhecimento humano, existe uma carência de estudos populacionais nos países em desenvolvimento, que investiguem a saúde mental dos idosos, principalmente aqueles residentes em municípios de pequeno porte.

Diante desta realidade, o objetivo do presente estudo foi estimar a prevalência e os fatores associados à TMC entre idosos de um município do Brasil. Os resultados do presente estudo podem contribuir para um maior entendimento da situação da saúde mental no Brasil, fornecendo subsídios para a criação de iniciativas de promoção e proteção da saúde mental dos idosos.

\section{Métodos}

Trata-se de um estudo epidemiológico de corte transversal, com dados extraídos do inquérito domiciliar intitulado "Monitoramento das Condições de Saúde de Idosos de um Município de Pequeno Porte (MONIDI)", realizado no município de Ibicuí, no Estado da Bahia, em 2014.

Ibicuí está localizada a $515 \mathrm{~km}$ de Salvador, capital da Bahia, no Sudoeste do estado, possui território de $1.176,843 \mathrm{Km}^{2}$, com 15.785 habitantes onde 2.125 são idosos, dentre os quais 525 estão cadastrados nas Unidades de Saúde da Família $(\mathrm{USF})^{13}$. Seu Índice de Desenvolvimento Humano Municipal (IDHM) é baixo $(0,584)$ sendo o $4540^{\circ}$ numa lista de 5.565 municípios brasileiros ${ }^{14}$.

Os critérios de inclusão utilizados no estudo foram: indivíduos com idade igual ou maior que 60 anos, residentes nas zonas urbana e rural, cadastrados pela USF do município de Ibicuí-BA. Foram excluídos todos os indivíduos com diagnóstico de demência ou qualquer outro tipo de alteração cognitiva que comprometesse a veracidade das informações fornecidas.

Após os critérios de exclusão, foi realizado um sorteio proporcional ao tamanho e distribuição por sexo e por USF, assumindo-se uma prevalência estimada de transtornos mentais comuns de $25 \%$, erro amostral de $3 \%$ e nível de confiança de $95 \%$.

Os participantes foram convidados a comparecer às USF nos dias de coleta, sendo informados sobre a pesquisa, sua importância e objetivos. Todos os entrevistados assinaram um Termo de Consentimento Livre e Esclarecido, comprometendo-se a participar voluntariamente deste estudo.

Como instrumento de coleta de dados, foi utilizado o Instrumento de avaliação da saúde de idosos (IASI), criado e validado previamente para utilização no presente estudo ${ }^{15}$. Este foi respondido pelos participantes na USF onde os usuários estavam cadastrados.

Para a avaliação da variável desfecho (TMC) foi utilizado o Self Reporting Questionnaire (SRQ-20), desenvolvido pela $\mathrm{OMS}^{16}$, validado e adaptado no Brasil por Gonçalves ${ }^{17}$. Na classificação de TMC, foi adotado o ponto de corte de cinco ou mais respostas positivas ${ }^{17,18}$.

Foram incluídas as variáveis independentes: Informações sociodemográficas (faixa etária: 60-79 anos/ $\geq 80$ anos, sexo: masculino/feminino, escolaridade: alfabetizado/não alfabetizado, renda: até um salário mínimo/acima de um salário mínimo, e situação conjugal: vive atualmente com companheiro/sem companheiro); Hábitos de vida (inatividade física no lazer: sim [não participa de no mínimo 150 minutos por sema- 
na de atividades físicas moderadas/intensas por semana no seu tempo livre] /não [participa de no mínimo 150 minutos de atividade física moderada/intensa por semana no tempo livre, fuma atualmente: sim/não, já foi fumante: sim/não, etilismo: sim [consome bebidas alcoólicas regularmente] /não [não consome bebidas alcoólicas regularmente] e tempo gasto sentado: acúmulo de até 260 minutos por semana ou $>260$ minutos por semana em atividades realizadas na posição sentada); Doenças referidas (asma/bronquite, hipertensão arterial sistêmica, hipercolesterolemia, reumatismo e dores na coluna), avaliadas por meio de questão dicotômica sim/não.

Os dados foram tabulados com auxílio do software EpiData ${ }^{19}$ versão 3.1, realizado o processo de dupla tabulação dos dados, no intuito de avaliar a qualidade dos dados. Inicialmente, foi feita análise descritiva considerando variáveis sociodemográficas, variáveis comportamentais, condições clínicas e saúde mental, com a finalidade de apresentar o perfil da população estudada.

Em seguida, foram calculadas razões de prevalência (RP) e seus respectivos intervalos de confiança a 95\% (IC = 95\%). Para avaliação da medida de significância estatística, utilizou-se o teste de qui-quadrado de Pearson, adotando $\alpha=5 \%$.

$\mathrm{A}$ análise de regressão de Poisson ${ }^{20}$ foi conduzida conforme os seguintes procedimentos: $1^{\text {a }}$ ) Seleção das variáveis a partir dos objetivos do estudo e da revisão da literatura; 2a) Verificação de pressupostos do modelo; 3a) Pré-seleção das variáveis (análise bivariada) para inclusão na análise através do teste de razão de verossimilhança, adotando-se um valor de $\left.\mathrm{p} \leq 0,20 ; 4^{\mathrm{a}}\right)$ Análise simultânea de todas as variáveis pré-selecionadas, com avaliação das medidas de associação com e sem a variável sob investigação, utilizando procedimento backward.

A análise dos dados foi realizada no pacote estatístico Statistical Package for Social Sciences - SPSS for Windows , versão 16.0.

O estudo foi submetido e aprovado pelo Comitê de Ética e Pesquisa da Universidade Estadual do Sudoeste da Bahia, de acordo com os princípios contidos na Declaração de Helsinque ${ }^{21}$, da Associação Médica Mundial, e pautados na resolução no 466/2012, do Conselho Nacional de Saúde (CNS).

\section{Resultados}

Foram considerados elegíveis para o estudo 335 idosos, dos quais 25 (7,46\%) foram considerados perdas e recusas, totalizando 310 participantes (taxa de respostas de 92,53\%) (Figura 1).

Entre os entrevistados, predominaram os indivíduos do sexo feminino (56,5\%), de faixa etária entre 60 e 79 anos (83,9\%), que viviam sem companheiro (51\%), com renda mensal de um salário mínimo ou menos $(93,2 \%)$, alfabetizados $(56,1 \%)$ que não consumiam bebidas alcoólicas regularmente $(95,8 \%)$, que relataram já terem fumado em algum momento da vida $(56,1 \%)$ e que não fumavam atualmente $(88,4 \%)$.

A maioria dos idosos era inativa fisicamente no lazer (69\%) e acumulavam até 260 minutos por semana em atividades realizadas na posição sentada $(57,7 \%)$. Entre as doenças referidas prevaleceram hipertensão arterial $(64,2 \%)$ e reumatismo $(31,6 \%)$.

A prevalência geral de TMC foi de $55,8 \%$. Entre as dimensões de TMC, os sintomas mais relatados foram assustar-se com facilidade $(57,4 \%) \mathrm{e}$ sentir-se nervoso, tenso ou preocupado (54,5\%), relacionados à dimensão humor depressivo $(\mathrm{Ta}-$ bela 1).

Identificou-se maior prevalência de TMC entre os idosos do sexo feminino $(66,9 \%, \mathrm{p}<$ $0,001)$, faixa etária $\geq 80$ anos $(62,0 \%, p=0,336)$, não alfabetizados $(61 \%, p=0,102)$, entre os que

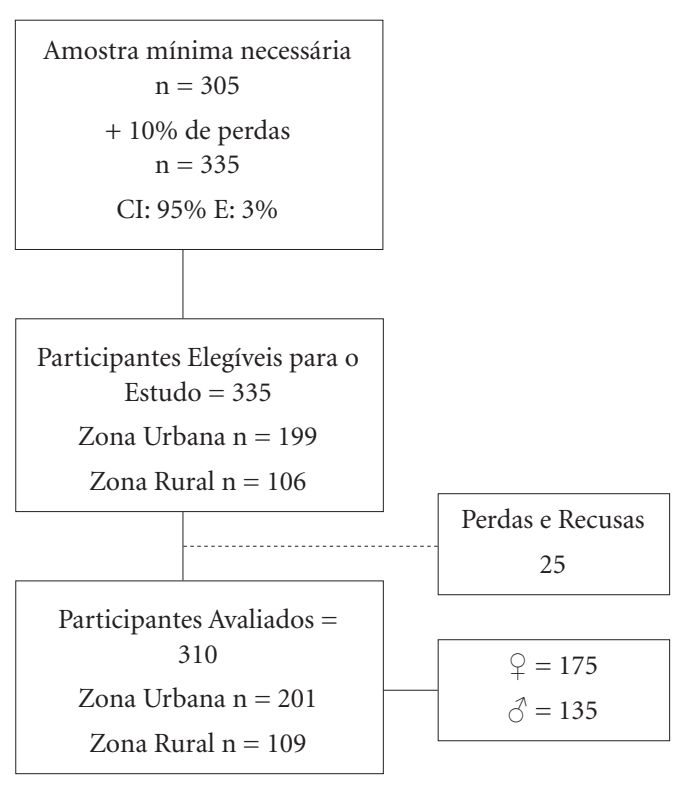

Figura 1. Esquema do processo de seleção e amostra do estudo. 
relataram renda de 1 salário mínimo ou menos =0,676). No entanto, a associação foi estatistica$(58,3 \%, \mathrm{p}=0,002)$ e aqueles que vivem sem com- mente significante apenas para as variáveis sexo e panheiros (separados/viúvos/solteiros) (57\%, p renda (Tabela 2).

Tabela 1. Prevalência de TMC segundo Self Reporting Questionnaire (SRQ-20). Ibicuí-BA, 2014.

\begin{tabular}{|c|c|c|}
\hline Dimensões de TMC & $\mathbf{N}$ & $\%$ \\
\hline \multicolumn{3}{|l|}{ Humor Depressivo } \\
\hline Assusta-se com facilidade & 178 & 57,4 \\
\hline Sente-se nervoso, tenso ou preocupado & 169 & 54,5 \\
\hline Tem se sentido triste ultimamente & 133 & 42,9 \\
\hline Tem chorado mais do que de costume & 76 & 24,5 \\
\hline \multicolumn{3}{|l|}{ Sintomas Somáticos } \\
\hline Tem dores de cabeça frequentemente & 103 & 33,2 \\
\hline Tem falta de apetite & 122 & 39,4 \\
\hline Dorme mal & 107 & 34,5 \\
\hline Tem tremores nas mãos & 50 & 16,5 \\
\hline Tem má digestão & 36 & 11,6 \\
\hline Tem sensações desagradáveis no estômago & 93 & 30 \\
\hline \multicolumn{3}{|l|}{ Decréscimo de Energia Vital } \\
\hline Tem dificuldade de pensar com clareza & 78 & 25,2 \\
\hline Encontra dificuldade de realizar com satisfação suas tarefas diárias & 90 & 29 \\
\hline Tem dificuldade em tomar decisões & 71 & 22,9 \\
\hline Seu trabalho diário lhe causa sofrimento & 48 & 15,5 \\
\hline Sente-se cansado o tempo todo & 76 & 24,5 \\
\hline Você se cansa com facilidade & 97 & 31,3 \\
\hline \multicolumn{3}{|l|}{ Pensamentos Depressivos } \\
\hline É incapaz de desempenhar um papel útil em sua vida & 73 & 23,5 \\
\hline Tem perdido o interesse pelas coisas & 87 & 28,1 \\
\hline Você se sente uma pessoa inútil em sua vida & 64 & 20,6 \\
\hline Tem tido ideia de acabar com sua vida & 12 & 3,9 \\
\hline
\end{tabular}

Tabela 2. Prevalência de TMC segundo características sociodemográficas. Ibicuí-BA, 2014.

\begin{tabular}{|c|c|c|c|}
\hline \multirow{2}{*}{ Variável } & \multicolumn{3}{|c|}{ Características sociodemográficas } \\
\hline & $\%$ & RP (IC 95\%) & Valor de $\mathrm{p}$ \\
\hline \multicolumn{4}{|l|}{ Sexo } \\
\hline Masculino & 41,5 & $0,62(0,44-0,73)$ & $<0,001$ \\
\hline Feminino & 66,9 & - & - \\
\hline \multicolumn{4}{|l|}{ Faixa Etária } \\
\hline$\geq 80$ anos & 62,0 & $1,13(0,82-1,74)$ & 0,336 \\
\hline $60-79$ anos & 54,6 & - & - \\
\hline \multicolumn{4}{|l|}{ Escolaridade } \\
\hline Não Alfabetizado & 61,0 & $1,17(0,95-1,60)$ & 0,102 \\
\hline Alfabetizado & 51,7 & - & - \\
\hline \multicolumn{4}{|l|}{ Renda Mensal } \\
\hline$\leq 1 \mathrm{SM}$ & 58,3 & $2,44(1,39-2,41)$ & 0,002 \\
\hline$>1 \mathrm{SM}$ & 23,8 & - & - \\
\hline \multicolumn{4}{|l|}{ Situação Conjugal } \\
\hline Sem companheiro & 57,0 & $1,05(0,74-1,22)$ & 0,676 \\
\hline Com companheiro & 54,6 & - & - \\
\hline
\end{tabular}

*Valor de p obtido por meio do Teste Qui-quadrado. 
A prevalência de TMC foi mais acentuada entre os indivíduos que referiram maior tempo gasto sentado $(62,6 \%, \mathrm{p}=0,039)$, inativos no lazer $(48,5 \%, \mathrm{p}=0,061)$, que consumiam bebidas alcoólicas regularmente $(61,5 \%, \mathrm{p}=0,680) \mathrm{e}$ aqueles que relataram fumar atualmente $(58,3 \%$, $\mathrm{p}=0,745)$ e entre os que relataram que já tinham fumado em algum período da vida $(56,1 \%, \mathrm{p}=$ 0,974), e a associação foi estatisticamente significante apenas para a variável tempo gasto sentado (Tabela 3).
Quando analisada a associação entre TMC e doenças referidas, identificou-se que os indivíduos que relataram ter asma/bronquite $(84,6 \%$, p $=0,033)$, hipertensão arterial sistêmica $(60,3 \%, \mathrm{p}$ $=0,33)$, hipercolesterolemia $(67,7 \%, p=0,005)$, reumatismo $(75,5 \%, \mathrm{p}=<0,001)$ e dores na coluna $(64,8 \%, p=<0,001)$. Identificou-se associação estatisticamente significante para as variáveis asma/bronquite, hipercolesterolemia, reumatismo e dores de coluna. (Tabela 4).

Tabela 3. Prevalência de TMC segundo hábitos de vida. Ibicuí-BA, 2014.

\begin{tabular}{|c|c|c|c|}
\hline \multirow{2}{*}{ Variável } & \multicolumn{3}{|c|}{ Hábitos de vida } \\
\hline & $\%$ & RP (IC 95\%) & Valor de p \\
\hline \multicolumn{4}{|l|}{ Inatividade física no lazer } \\
\hline Sim & 48,5 & $0,81(0,62-1,02)$ & 0,061 \\
\hline Não & 59,2 & - & - \\
\hline \multicolumn{4}{|l|}{ Fuma atualmente } \\
\hline Sim & 58,3 & $1,05(0,62-1,41)$ & 0,745 \\
\hline Não & 55,5 & - & - \\
\hline \multicolumn{4}{|l|}{ Já foi fumante } \\
\hline Sim & 56,1 & $1,00(0,77-1,28)$ & 0,974 \\
\hline Não & 55,9 & - & - \\
\hline \multicolumn{4}{|l|}{ Etilismo } \\
\hline Sim & 61,5 & $1,10(0,43-1,75)$ & 0,680 \\
\hline Não & 55,7 & - & - \\
\hline \multicolumn{4}{|l|}{ Tempo gasto sentado } \\
\hline$>260$ minutos/semana & 62,6 & $1,23(1,01-1,72)$ & 0,039 \\
\hline$\leq 260$ minutos/semana & 50,8 & - & - \\
\hline
\end{tabular}

${ }^{*}$ Valor de p obtido por meio do Teste Qui-quadrado.

Tabela 4. Prevalência de TMC segundo doenças referidas. Ibicuí, Bahia, 2014.

\begin{tabular}{|c|c|c|c|}
\hline \multirow{2}{*}{ Variável } & \multicolumn{3}{|c|}{ TMC } \\
\hline & $\%$ & RP (IC 95\%) & Valor de $\mathrm{p}$ \\
\hline \multicolumn{4}{|l|}{ Asma/Bronquite } \\
\hline Sim & 84,6 & $1,55(0,82-10,6)$ & 0,033 \\
\hline Não & 54,5 & - & - \\
\hline \multicolumn{4}{|l|}{ Hipertensão Arterial Sistêmica } \\
\hline $\operatorname{Sim}$ & 60,3 & $1,26(1,03-1,68)$ & 0,33 \\
\hline Não & 47,7 & - & - \\
\hline \multicolumn{4}{|l|}{ Hipercolesterolemia } \\
\hline Sim & 67,7 & $1,34(1,11-2,11)$ & 0,005 \\
\hline Não & 50,5 & - & - \\
\hline \multicolumn{4}{|l|}{ Reumatismo } \\
\hline Sim & 75,5 & $1,61(1,50-3,15)$ & $<0,001$ \\
\hline Não & 46,7 & - & - \\
\hline \multicolumn{4}{|l|}{ Dores na Coluna } \\
\hline Sim & 64,8 & $1,63(1,35-2,18)$ & $<0,001$ \\
\hline Não & 39,6 & - & - \\
\hline
\end{tabular}

* Valor de p obtido por meio do Teste Qui-quadrado. 
Após analise de regressão de Poisson as variáveis sexo e reumatismo, mantiveram associação com TMC. Os indivíduos do sexo feminino e que referiram reumatismo continuaram apresentando maiores prevalências de TMC (Tabela 5).

\section{Discussão}

A prevalência geral de TMC encontrada neste estudo foi mais elevada quando comparada a outras localidades ${ }^{3,6,9,11,22}$, caracterizando-se como um sério problema de saúde pública.

Recentes estudos em diferentes regiões brasileiras apresentam valores discrepantes quanto à prevalência de TMC entre idosos. Observou-se prevalências maiores em municípios como Jequi-

Tabela 5. Razão de prevalência e intervalos de confiança bruta e ajustada entre a prevalência de TMC e variáveis do estudo. Ibicuí, Bahia, 2014.

\begin{tabular}{|c|c|c|}
\hline Variável & $\begin{array}{l}\text { RP e IC } \\
(95 \%)_{\text {Bruta }}\end{array}$ & $\begin{array}{c}\text { RP e IC } \\
(95 \%)_{\text {Ajustada }}\end{array}$ \\
\hline \multicolumn{3}{|l|}{ Sexo } \\
\hline Masculino & 1,00 & 1,00 \\
\hline Feminino & $0,62(0,44-0,73)$ & $1,51(1,08-2,12)$ \\
\hline \multicolumn{3}{|l|}{ Renda } \\
\hline$\leq 1 \mathrm{SM}$ & 1,00 & 1,00 \\
\hline$>1 \mathrm{SM}$ & $2,44(1,39-2,41)$ & $0,55(0,22-1,36)$ \\
\hline \multicolumn{3}{|l|}{ Escolaridade } \\
\hline Não alfabetizado & 1,00 & 1,00 \\
\hline Alfabetizado & $1,17(0,95-1,60)$ & $0,84(0,62-1,14)$ \\
\hline \multicolumn{3}{|l|}{ Tempo gasto sentado } \\
\hline$>260$ minutos/semana & 1,00 & 1,00 \\
\hline$\leq 260$ minutos/semana & $1,23(1,01-1,72)$ & $0,79(0,58-1,07)$ \\
\hline \multicolumn{3}{|l|}{ Inatividade física no lazer } \\
\hline Sim & 1,00 & 1,00 \\
\hline Não & $0,81(0,62-1,02)$ & $0,97(0,68-1,36)$ \\
\hline \multicolumn{3}{|l|}{ Asma/Bronquite } \\
\hline Sim & 1,00 & 1,00 \\
\hline Não & $1,55(0,82-10,6)$ & $0,73(0,40-1,36)$ \\
\hline \multicolumn{3}{|l|}{ Hipercolesterolemia } \\
\hline Sim & 1,00 & 1,00 \\
\hline Não & $1,34(1,11-2,11)$ & $0,98(0,69-1,38)$ \\
\hline \multicolumn{3}{|l|}{ Reumatismo } \\
\hline $\operatorname{Sim}$ & 1,00 & 1,00 \\
\hline Não & $1,61(1,50-3,15)$ & $0,68(0,49-0,95)$ \\
\hline \multicolumn{3}{|l|}{ Dores na coluna } \\
\hline Sim & 1,00 & 1,00 \\
\hline Não & $1,63(1,35-2,18)$ & $0,75(0,49-0,95)$ \\
\hline
\end{tabular}

é-BA $(47,4 \%)^{9}$ e Rio de Janeiro-RJ $(43,6 \%)^{22}$. Em outros estudos brasileiros a ocorrência de TMC foi mais baixa, como em Feira de Santana-BA $(29,6 \%)^{8}$, Campinas-SP $(29,7 \%)^{11}$ e Catalão-GO $(31,47 \%)^{4}$. No cenário internacional, a prevalência de TMC entre idosos variou de $32,4 \%{ }^{6}$, na Etiópia, a 51,8\%, na Dinamarca ${ }^{7}$.

Entre os sintomas de TMC, os mais relatados foram relacionados à dimensão humor depressivo (assusta-se com facilidade e sente-se nervoso, tenso ou preocupado). Estudos realizados com idosos também observaram resultado semelhante, mostrando que esses sintomas parecem ser mais prevalentes entre os idosos ${ }^{4,11}$.

A exposição à TMC é mais acentuada durante o processo de envelhecimento. Diversos fatores podem estar relacionados a esse quadro, dentre eles a elevada presença de comorbidades e incapacidades, e condições precárias de vida ${ }^{8,11}$, episódios de estresse durante a vida e isolamento social ${ }^{11}$.

Além da prevalência elevada, observou-se no presente estudo que a probabilidade de apresentar TMC foi maior entre os indivíduos do sexo feminino. Tal fato pode ser atribuído a sobrecarga nas atividades exercidas pela mulher na sociedade, materializadas na sobreposição de tarefas oriundas do mercado de trabalho, atividades domésticas e de cuidado com a educação dos filhos ${ }^{4,8,23}$, além da desvalorização e violência a qual está exposta esta população.

Essa situação pode levar a situações de tristeza profunda, ansiedade, frustração, angustia e adoecimento, aumentando a exposição a $\mathrm{TMC}^{4,23}$. Ainda, nota-se que a população feminina apresenta maior facilidade de identificar o adoecimento, relatam melhor os sintomas de doenças, além de buscarem com mais frequência os serviços de saúde ${ }^{4}$.

Após a análise multivariada, o reumatismo foi a única doença referida que se manteve associada à TMC. As morbidades em geral, aumentam a possibilidade dos indivíduos apresentarem incapacidades ${ }^{24}$. A incapacidade funcional, o aumento da vulnerabilidade e a dependência, são fatores que se relacionam a presença de doenças crônicas, fato este que leva ao isolamento social e, consequentemente, eleva a possibilidade de apresentar sintomas somáticos, depressivos e ansiosos, entre outros ${ }^{11}$.

Dentre as limitações do estudo, pode-se destacar o delineamento metodológico, visto que um estudo de corte transversal não permite a avaliação da causa e efeito entre as variáveis estudadas. Por outro lado, até onde se sabe, este é o primeiro estudo populacional que rastreou 
TMC entre idosos de um município com baixo IDH, utilizando como instrumento de triagem o SRQ-20, instrumento indicado pela OMS para levantamento sobre a saúde mental em países em desenvolvimento.

Além disso, o estudo foi conduzido com uma população pouco estudada no Brasil, sendo possível gerar informações úteis e válidas que podem contribuir para as políticas de atenção à saúde e garantia de qualidade de vida e para orientar medidas de intervenção em saúde direcionadas a esse grupo populacional, que se encontra em contínuo crescimento no país.

\section{Colaboradores}

PAS Silva trabalhou na coleta de dados, delineamento metodológico, redação do artigo, interpretação dos dados e aprovação da versão a ser publicada; SV Rocha trabalhou na concepção, delineamento metodológico, análise dos dados, revisão crítica e aprovação da versão a ser publicada; LB Santos trabalhou no delineamento metodológico, interpretação dos dados, revisão crítica do conteúdo e aprovação da versão a ser publicada; CA Santos, CR Amorim e ABA Vilela trabalharam na concepção, delineamento metodológico, revisão crítica do conteúdo e aprovação da versão a ser publicada.

\section{Agradecimentos}

Ao Conselho Nacional de Desenvolvimento Científico e Tecnológico (CNPq) pela concessão da bolsa de iniciação científica e ao Núcleo de Estudo em Saúde da População (NESP) pela contribuição ao ceder os dados da pesquisa para a realização deste artigo. 


\section{Referências}

1. Knudsen AK, Harvey SB, Mycletun A, Overland S. Common mental disorders and long-term sickness absence in a general working population. The H H Study. Acta Psychiatr Scand 2013; 127(4):287-297.

2. Skapinakis P, Bellos S, Koupidis S, Grammatikopoulos L, Theodorakis PN, Mavreas V. Prevalence and sociodemographic associations of CMD in a nationally representative sample of the general population of Greece. BMC Psychiatry 2013; 13:163.

3. Goldberg D, Huxley P. Common mental disorders: a bio-social model. London: Tavistock; 1992.

4. Lucchese R, Sousa K, Bonfin SP, Vera I, Santana FR. Prevalência de transtorno mental comum na atenção primária. Acta Paul de Enferm 2014; 27(3):200-207.

5. Gois RO, Gois BO, Pereira MCCS, Taguchi CK. Estado mental e impacto do zumbido em idosos. Rev CEFAC 2014; 16(3):798-809.

6. Yimam K, Kebede Y, Azale T. Prevalence of Common Mental Disorders and Associated Factors among Adults in Kombolcha Town, Northeast Ethiopia. J Depress Anxiety 2014; 1(7):2167-1044.

7. Soegaard HJ, Pedersen P. Prevalence of Common Mental Disorders among Incident Individuals on Long-Term Sickness Absence When Compensating for Non-Participation. Psychology 2012; 3(9A):818-824.

8. Rocha SV, Almeida MMG, Araujo TM, Rodrigues WKM, Santos LB, Junior JSV. Prevalência de transtornos mentais comuns entre idosos residentes em município do Nordeste do Brasil. Rev de Salud Pública 2012; 14(4):620-629.

9. Pinto LLT, Rocha SV, Viana HPS, Rodrigues WKM, Vasconcelos LRC. Nível de atividade física habitual e transtornos mentais comuns entre idosos residentes em áreas rurais. Rev Bras de Geriatr e Gerontol 2014; 17(4):819-828.

10. Rocha SV, Almeida MMG, Araujo TM, Junior JSV. Atividade física no lazer e transtornos mentais comuns entre idosos residentes em um município do nordeste do Brasil. J Bras de Psiquiatr 2011; 60(2):80-85.

11. Borim FSA, Barros MBA, Botega NJ. Transtorno mental comum na população idosa: pesquisa de base populacional no Município de Campinas, São Paulo, Brasil. Cad Saude Publica 2013; 29(7):1415-1426.

12. Santos LM, Cortina I. Fatores que contribuem para a depressão no idoso. Rev Enferm UNISA 2011; 12(2): 112-116.

13. Instituto Brasileiro de Geografia e Estatística (IBGE). Censo Demográfico 2010: resultados preliminares da amostra. Rio de Janeiro: IBGE; 2011.

14. Atlas do Desenvolvimento Humano no Brasil 2013. Ranking Bahia, 2010. [acessado 2014 Set 25]. Disponível em: http://www.atlasbrasil.org.br/2013/pt/ranking.

15. Reis MC, Nascimento RAS, Pedreira RBS, Rocha SV, Vasconcelos LRC, Diniz KO, Santos CA. Validação de face e clareza do Instrumento de Avaliação da Saúde dos Idosos - IASI. In: Anais do XIX Congresso Brasileiro de Geriatria e Gerontologia; 2014; Belém. p. 296.
16. Organização Mundial Da Saúde (OMS). Relatório Mundial da Saúde. Saúde Mental: Nova concepção, nova esperança. Lisboa: OMS; 2002.

17. Gonçalves DM, Stein AT, Kapczinski F. Avaliação de desempenho do Self-Reporting Questionnaire como instrumento de rastreamento psiquiátrico: um estudo comparativo com o Structured Clinical Interview for DSM-IV-TR. Cad Saude Publica 2008; 24(2):380-390.

18. Scazufca M, Menezes PR, Vallada H, Araya R. Validity of the Self-Reporting Questionnaire-20 in epidemiological studies with older adults. Soc Psychiatry Psychiatr Epidemiol 2009; 44(3):247-254.

19. Lauritsen JH, Bruss M, Myatt MA. Epidata [software]. Programa para criar banco de dados. Odense: EpiData Association; 2002. (v3.0). Versão para o português (Brasil) por João Paulo Amaral Haddad.

20. Barros AJ, Hirakata VN. Alternatives for logistic regression in cross-sectional studies: an empirical comparison of models that directly estimate the prevalence ratio. BMC Med Res Methodol 2003; 3:21.

21. Declaração de Helsinque. Princípios éticos para as pesquisas médicas em seres humanos. Associação Médica Mundial: 52a Assembléia Geral; 2000 Out; Edimburgo, Escócia. [acessado 2015 Ago 19]. Disponível em: http:// www.fcm.unicamp.br/fcm/sites/default/files/declaracao_de_helsinque.pdf

22. Martins LCX, Kuhn L. Prevalência de transtornos mentais comuns em jovens brasileiros recém-incorporados ao Serviço Militar Obrigatório e fatores associados. Cien Saude Colet 2013; 18(6):1809-1816.

23. Rocha SV, Almeida MMG, Araujo TM, Junior JSV. Prevalência de transtornos mentais comuns entre residentes em áreas urbanas de Feira de Santana, Bahia. Rev Bras Epidemiol 2010; 13(4):630-640.

24. Virtuoso Júnior S, Guerra RO. Incapacidade funcional em mulheres idosas de baixa renda. Cien Saude Colet 2011; 16(5):2541-2548.

Artigo apresentado em 25/11/2015

Aprovado em 18/05/2016

Versão final apresentada em 20/05/2016 